\title{
CN as a tracer of galaxy assembly timescales
}

\author{
C. Carretero ${ }^{1}$, A. Vazdekis ${ }^{1}$, J.E. Beckman ${ }^{1,2}$, P. Sánchez-Blázquez ${ }^{3}$ \\ and J. Gorgas ${ }^{3}$ \\ ${ }^{1}$ Instituto de Astrofisica de Canarias. Vía Láctea s/n. 38200 La Laguna, Tenerife, Spain. \\ email: cch@iac.es \\ ${ }^{2}$ Consejo Superior de Investigaciones Científicas, Spain. \\ ${ }^{3}$ Universidad Complutense de Madrid. Ciudad Universitaria, Madrid, Spain.
}

\begin{abstract}
We present the values of $\mathrm{CN}$ and $\mathrm{Mg}$ overabundances with respect to Fe, for a large sample of elliptical galaxies in different environments. Abundances were derived by confronting observed absorption line indices with stellar population model spectra. We obtained significant differences between the $[\mathrm{CN} / \mathrm{Fe}]$ and $[\mathrm{Mg} / \mathrm{Fe}]$ abundance ratios as a functions of: i) the environment, and ii) the galaxy mass. This is interpreted as implying varying formation timescales for $\mathrm{CN}, \mathrm{Mg}$ and $\mathrm{Fe}$, combined with different star formation histories in elliptical galaxies depending on their mass and environment. Our principal conclusions are: 1) CN is sensitive to the characteristic assembly timescales of elliptical galaxies, 2) more massive elliptical galaxies are assembled on shorter timescales than less massive ones, 3) elliptical galaxies in denser environments are assembled on shorter timescales than those in lower density environments, and 4) our results strongly suggest an upper limit for the assembly timescale of $\sim 1 \mathrm{~Gy}$, in all cases.
\end{abstract}

Keywords. Cosmology: observations, galaxies: abundances, galaxies: clusters: general, galaxies: formation, galaxies: stellar content, X-rays: galaxies: clusters

\section{Introduction}

Stellar populations offer a fossil record of the formation and evolution ofgalaxies, providing very strong constraints on the principal galaxy formation scenarios. So, a programme to understand stellar populations as a function of the environment will give many answers to the puzzle. Differences in the abundance of $\mathrm{CN}, \mathrm{Mg}$ and $\mathrm{Fe}$, as a function of the environment, have been recently suggested by Sánchez-Blázquez et al. (2003). We explore these differences by studying a number of galaxy clusters.

\section{Abundance estimation and results}

To derive mean luminosity-weighted ages and metallicities, we compared selected absorption line strength indices $\left[\mathrm{CN}_{2}, \mathrm{Mg}_{2}\right.$ and $\mathrm{Fe} 2$ (Worthey 1994)] with those predicted by the model of Vazdekis et al. (1999). This model provides flux-calibrated spectra at a resolution of $1.8 \AA$ (FWHM) for single-burst stellar populations. Plots of the strengths of the indices versus $\mathrm{H}_{\beta}$ provide rather orthogonal model grids, allowing us to accurately estimate galaxy mean ages as well as the abundances of these elements. Finally, we determined the overabundances of each cluster, as the mean values of the overabundances of all the galaxies within it. Errors were computed as the standard deviation.

We used X-ray luminosity as a quantitative indicator of the mass of the clusters. We adopted X-ray luminosity values from Ebeling et al. (1998) and Ledlow et al. (2003). Figure 1 shows the values of $[\mathrm{CN} / \mathrm{Fe}],[\mathrm{Mg} / \mathrm{Fe}]$ and $[\mathrm{Mg} / \mathrm{CN}]$ versus X-ray luminosity for each cluster. We found clear correlations between $[\mathrm{CN} / \mathrm{Fe}]$ and $[\mathrm{Mg} / \mathrm{CN}]$ values and X-ray luminosity. No correlation was found for $[\mathrm{Mg} / \mathrm{Fe}]$. 


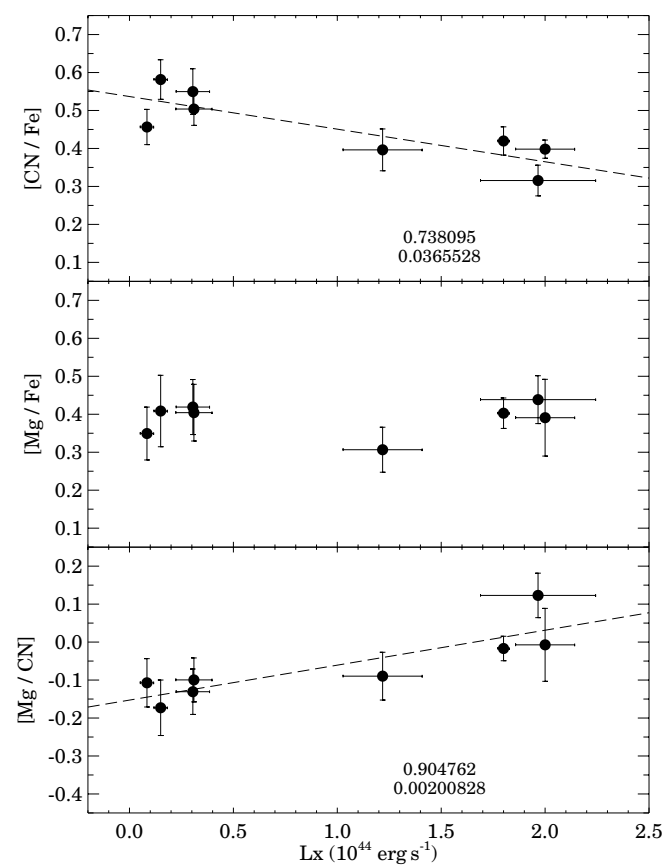

Figure 1. Cluster X-ray luminosity vs. overabundance values of $[\mathrm{CN} / \mathrm{Fe}]($ top $),[\mathrm{Mg} / \mathrm{Fe}]$ (middle) and $[\mathrm{Mg} / \mathrm{CN}]$ (bottom). Each point corresponds to one individual cluster, and is computed as the mean value of the overabundaces of early-type galaxies within each cluster, with $150 \mathrm{~km} \mathrm{~s}^{-1} \leqslant \sigma \leqslant 250 \mathrm{~km} \mathrm{~s}^{-1}$. The correlation coefficient and its significance are written in top and bottom panels. Taken from Carretero et al. (2004).

\section{Discussion}

The correlations are interpreted in terms of the different formation timescales for each element, and the different SFH of early-type galaxies, as a function of their environment.

The constancy of the $[\mathrm{Mg} / \mathrm{Fe}]$ overabundance is interpreted in terms of the great difference in the formation timescales of the two elements: the galaxues are fully assembled before SNeIa can significantly pollute with Fe the ISM of the smaller galaxies before merging, and right after $\mathrm{Mg}$ is fully ejected. Since $[\mathrm{Mg} / \mathrm{Fe}]$ is found to be constant with $\mathrm{X}$-ray luminosity of the clusters, which is an indicator of their mass, we conclude that this ratio is independent of the environment. Similar results for the $[\mathrm{Mg} / \mathrm{Fe}]$ ratio have been obtained by other authors, as Sánchez-Blázquez et al. (2003).

But, when considering species with not so different formation timescales, such as $\mathrm{CN}$ and $\mathrm{Fe}$, or $\mathrm{CN}$ and $\mathrm{Mg}$, clear correlations are found between abundance ratios and the environment. The fact that $[\mathrm{CN} / \mathrm{Fe}]$ decreases with the cluster X-ray luminosity, and that $[\mathrm{Mg} / \mathrm{CN}]$ increases with it, suggests that galaxies in more massive clusters are fully assembled on shorter timescales than those in less massive clusters.

\section{References}

Carretero, C., Vazdekis, A., Beckman, J.E., Sánchez-Blázquez, P., \& Gorgas, J. 2004, ApJ, 609, L45

Ebeling, H. et al. 1998, MNRAS, 301, 881

Ledlow, M.J., Voges, W., Owen, F.N., \& Burns, J.O. 2003, AJ, 126, 2740

Sánchez-Blázquez, P., Gorgas, J., Cardiel, N., Cenarro, J., \& González, J.J. 2003, ApJ, 590, L91

Vazdekis, A. 1999, ApJ, 513, 224

Worthey, G. 1994, ApJS, 95, 107 